\title{
Isolation and Structural Analysis In Vivo of Newly Synthesized Fructooligosaccharides in Onion Bulbs Tissues (Allium cepa L.) during Storage
}

\author{
Masaki Fujishima, ${ }^{1}$ Kensuke Furuyama, ${ }^{1}$ Yojiro Ishihiro, ${ }^{1}$ Shuichi Onodera, ${ }^{1}$ Eri Fukushi, ${ }^{2}$ \\ Noureddine Benkeblia, ${ }^{3}$ and Norio Shiomi ${ }^{1}$ \\ ${ }^{1}$ Department of Food Science, Graduate School of Dairy Science Research, Rakuno Gakuen University, Ebetsu 069-8501, Japan \\ ${ }^{2}$ Graduate School of Agriculture, Hokkaido University, Sapporo 060-8589, Japan \\ ${ }^{3}$ Department of Life Sciences, The University of the West Indies, Mona Campus, Kingston 7, Jamaica \\ Correspondence should be addressed to Norio Shiomi, n-shiomi@rakuno.ac.jp
}

Received 2 February 2009; Accepted 20 April 2009

Recommended by Thomas Heinze

Fructooligosaccharides are involved in physiological activities and quality attributes of onion bulbs. This work describes structures of newly synthesized oligosaccharides formed by fructose moieties in onion bulb tissues during storage. Onion bulbs were stored for four weeks at $10^{\circ} \mathrm{C}$. HPAEC-PAD analysis showed that saccharide 1 was eluted after 1-kestose while saccharide 2 was eluted after nystose (4a). Saccharides 1 and 2 have R-sucrose values of 1.55 and 2.15 by HPAEC, a reducing terminal, a reducing sugar-to-fructose ratio of 0.5 and 0.3 , and a degree of polymerization of 2 and 3 by TOF-MS, respectively. GLC analysis of the methyl derivatives and NMR measurement of the saccharides confirmed the presence of two different structures: the structure of saccharide 1 is composed by two fructose moieties and linked by $\beta(2 \rightarrow 1)$ linkage and was identified as inulobiose [ $\beta$-Dfructofuranosyl- $(2 \rightarrow 1)-\beta$-D-fructopyranose]. The structure of saccharide 2 consists of three units of fructose linked by $\beta(2 \rightarrow 1)$ linkage and was identified as inulotriose $[\beta$-D-fructofuranosyl- $(2 \rightarrow 1)-\beta$-D-fructofuranosyl- $(2 \rightarrow 1)-\beta$-D-fructopyranose]. The spectra also showed that 70 to $80 \%$ of the terminal fructose residue of the two saccharides is of pyranosyl form, while 20 to $30 \%$ is of furanosyl form. This finding demonstrated that these newly produced saccharides, catalyzed by onion-purified 6G-FFT, were synthesized by the action of 1-FFT fructosyltransfer from 1-kestose to free fructopyranose yielding inulobiose and sucrose, while elongation of fructofuranosyl units occurs at this transferred fructofuranosyl residue to produce inulooligosaccharide having an additional unit of fructofuranose.

Copyright ( 2009 Masaki Fujishima et al. This is an open access article distributed under the Creative Commons Attribution License, which permits unrestricted use, distribution, and reproduction in any medium, provided the original work is properly cited.

\section{Introduction}

About $80 \%$ of onion bulb (Allium cepa) dry matter consists of nonstructural carbohydrates [1]. The predominant of these nonstructural carbohydrates consist of glucose, fructose, sucrose, and low-molecular-weight fructooligosaccharides $[1,2]$. Fructans, fructooligosaccharides (FOSs) and polyfructosylsucroses of varying molecular size, are the main carbohydrate reserve in onion, as well as in other vegetative organs and plants including alliaceous organs (bulbs). Fructans accumulate during bulbing, and are then catabolized during the regrowth and the sprout development of the bulbs [3].
1-Kestose [3a, $1^{\mathrm{F}}-\beta$-D-fructofuranosylsucrose, 1-kestotriose $]$, Neokestose $\left[3 \mathbf{b}, 6^{\mathrm{G}}-\beta\right.$-D-fructofuranosylsucrose, $6 \mathrm{G}$-kestotriose $]$, nystose $\left[4 \mathrm{a}, 1^{\mathrm{F}} \text { (1- } \beta \text {-D-fructofuranosyl }\right)_{2}$ sucrose, 1,1 -kestotetraose $], 4 \mathbf{b}$ [6 $6^{\mathrm{G}}(1-\beta \text {-D-fructofuranosyl })_{2}$ sucrose, $1,6 \mathrm{G}$-kestotetraose $]$, and $4 \mathrm{c}\left[1^{\mathrm{F}}, 6^{\mathrm{G}}\right.$-di- $\beta$-fructofuranosyl sucrose, 1 and 6G-kestotetraose] constitute part of the different FOS found in onion bulb tissues and their contents vary between 4 and 5\%, 1.5 and $2.5 \%, 1.5$ and $2 \%, 4$ and $4.5 \%$, as well as 3 and $4 \%$ of total carbohydrates for DP $3 a, 3 b, 4 a, 4 b$, and $4 c$, respectively [2-5]. During the past decade, FOS have received considerable interest as food ingredients. They are used as nondigestible dietary fiber and texturing properties in many food stuff $[6-8]$. 
Some studies have been focused on the FOS properties with respect to their polydispersities, and few take into account their degradation parameters in vitro $[9,10]$ but only two referenced works have reported their in vivo hydrolysis parameters during the storage of the bulbs under different temperature regimes $[11,12]$.

During the postharvest life and the onset of sprouting, which is characterized by a catabolism of the FOS reserve, the activities of fructan:fructan 1-fructosyltransferase (1-FFT, EC 2.4.1.100) and fructan:fructan $6^{\mathrm{G}}$-fructosyltransferase (6G-FFT) involved in fructan biosynthesis were also observed simultaneously [13]. These two activities link one fructosyl residue by $\beta(2 \rightarrow 1)$ and $\beta(2 \rightarrow 6)$ initiating the formation of inulins and inulin neoseries FOS, respectively. Indeed, the involvement of two different enzymatic proteins (6G-FFT and 1-FFT) has been established in other vegetables, that is, asparagus, however, recent proteomic and genomic studies carried out on 6G-FFT and 1-FFT activities have demonstrated that both activities in onion bulbs are assigned to a single and unique enzymatic protein $[14,15]$.

Although it was established that net fructan accumulation only occurs when the rate of synthesis exceeds the rate of degradation in the vacuole [16], an imbalance occurs during the postharvest life which lead to the exhausting of the FOS reserve in onion tissues. Beside these biosynthetic activities, up to this date, no work reported the biosynthesis of new FOS in onion bulbs during storage, or in any other liliaceous plants as well. However, Van den Ende et al. [17] first succeeded to synthesize in vitro new fructosyl-only oligosaccharides using purified FFT of chicory roots. Later, Timmermans et al. [18] confirmed the presence of three new series of oligosaccharides in forced chicory roots (one disaccharide, one trisaccharide, and one tetrasaccharide) which contain neither $\beta(2 \rightarrow 1)$ nor $\beta(2 \rightarrow 6)$ linkages. Ernst et al. [19] reported later the presence of two inulo-n-ose series without a terminal glucose from species of the Allium genus.

The present work is devoted to the study of the isolation and structural analysis of newly formed short-chain FOS in stored onion bulbs.

\section{Materials and Methods}

2.1. Materials. Onion bulbs (Allium cepa cv. Tenshin, summer cultivar), which had been freshly harvested and dried (cured) in the field for two weeks, were obtained from the local farm of Rakuno Gakuen University, Ebetsu, Hokkaido, Japan. The bulbs were sorted for uniformity and absence of defects, and then packed in commercial plastic (PVC) trays of $10 \mathrm{~kg}$ each. Five trays were kept in store as described in what follows. FOS were analyzed immediately after harvesting and one month later in onion bulbs stored at $10^{\circ} \mathrm{C}$ and $70 \% \mathrm{RH}$.

2.2. Fructooligosaccharides Standards. 1 -Kestose $\left[3 a, 1^{\mathrm{F}}-\beta\right.$ D-fructofuranosylsucrose; 1 kestotriose] and nystose [4a, $1^{\mathrm{F}}(1-\beta \text {-D-fructofuranosyl })_{2}$ sucrose; 1,1 kestotetraose] were previously prepared in our laboratory as described by Takeda et al. [20]. Neokestose [3b: $6^{\mathrm{G}}-\beta$-D-fructofuranosylsucrose;
6G-kestotriose], $4 \mathbf{b}\left[6^{\mathrm{G}}\right.$ (1- $\beta$-D-fructofuranosyl) 2 sucrose; $1,6 \mathrm{G}$-kestotetraose ], $4 \mathrm{c}\left[1^{\mathrm{F}}, 6^{\mathrm{G}}\right.$-di- $\beta$-D-fructofuranosyl sucrose; 1 and $6 \mathrm{G}$-kestotetraose], 5a [1 $1^{\mathrm{F}}(1-\beta$-D-fructofuranosyl $)_{3}$ sucrose; $1,1,1$-kestopentaose $], 5 \mathbf{b}$ [6 ${ }^{\mathrm{G}}$ (1- $\beta$-D-fructofuranosyl $)_{3}$ sucrose; 1,1,6G-kestopentaose], $5 \mathrm{c}$ [1 $1^{\mathrm{F}}(1-\beta-\mathrm{D}$ fructofuranosyl $)_{2}-6^{\mathrm{G}}-\beta$-D-fructofuranosyl sucrose; 1,1 and 6G-kestopentaose], 5d [ $1^{\mathrm{F}}-\beta$-D-fructofuranosyl- $6^{\mathrm{G}}(1-\beta-\mathrm{D}$ fructofuranosyl $)_{2}$ sucrose; 1 and 1,6G-kestopentaose], $6 \mathbf{b}\left[6^{\mathrm{G}}\right.$ $(1-\beta \text {-D-fructofuranosyl })_{4}$ sucrose; $1,1,1,6 \mathrm{G}$-kestohexaose], 6c $\left[1^{\mathrm{F}}(1-\beta \text {-D-fructofuranosyl })_{3}-6^{\mathrm{G}}-\beta\right.$-D-fructofuranosyl sucrose; $1,1,1$ and $6 \mathrm{G}$-kestohexaose $], \mathbf{6 d}_{1}$ [1 ${ }^{\mathrm{F}}-\beta$-D-fructofuranosyl- $^{\mathrm{G}}$ (1- $\beta$-D-fructofuranosyl) 3 sucrose; 1 and $1,1,6 \mathrm{G}$ kestohexaose], $\mathbf{d d}_{2}$ [ $1^{\mathrm{F}}\left(1-\beta\right.$-D-fructofuranosyl) ${ }_{2} 6^{\mathrm{G}}(1-\beta-\mathrm{D}$ fructofuranosyl) $)_{2}$ sucrose; 1,1 and $1,6 \mathrm{G}$-kestohexaose], 7 [1 $^{\mathrm{F}}(1-\beta \text {-D-fructofuranosyl })_{m}-6^{\mathrm{G}}(1-\beta \text {-D-fructofuranosyl })_{n}$ sucrose $(m+n=5)], \mathbf{8}\left[1^{\mathrm{F}}\left(1-\beta\right.\right.$-D-fructofuranosyl) ${ }_{m}-6^{\mathrm{G}}(1-$ $\beta$-D-fructofuranosyl $)_{n}$ sucrose $\left.(m+n=6)\right]$, and $\mathrm{X} \geq 9$ [1 $^{\mathrm{F}}(1-\beta \text {-D-fructofuranosyl })_{m}-6^{\mathrm{G}}(1-\beta \text {-D-fructofuranosyl })_{n}$ sucrose $(m+n \geq 7)$ ] were obtained from asparagus roots as described in previous papers [21-23]. The standards $6 \mathbf{a}\left[1^{\mathrm{F}} \quad(1-\beta \text {-D-fructofuranosyl })_{4}\right.$ sucrose; $1,1,1,1$ kestohexaose $], 7 \mathbf{a}\left[1^{\mathrm{F}}(1-\beta \text {-D-fructofuranosyl })_{5}\right.$ sucrose; $1,1,1,1,1$-kestoheptaose] were prepared from Jerusalem artichoke tubers in our laboratory. Because the fructan nomenclature is not simple since the structures are very complex, the nomenclatures for FOS proposed by Lewis [24] and Waterhouse and Chatterton [25] were used. All isolated and synthesized standards are of high-grade purity $(\geq 99.8 \%)$.

2.3. Fructooligosaccharides Extraction. Fructooligosaccharides (FOSs) were extracted by the method of Shiomi [26]. Tissues ( $10 \mathrm{~g}$ ) were homogenized in $80 \mathrm{~mL}$ of aqueous ethanol $(70 \%)$ using a small amount of calcium carbonate $\left(0.5 \mathrm{~g} \mathrm{~L}^{-1}\right)$. The homogenate was boiled under reflux in a water bath for 10 minutes. Homogenate was filtered and the residue extracted three times with aqueous ethanol and one time with water in the same conditions. The filtrates were combined and made up to $500 \mathrm{~mL}$ with distilled water. An aliquot of the filtrate $(10 \mathrm{~mL})$ was concentrated to dryness under vacuum at $35^{\circ} \mathrm{C}$ using a Büchi rotavapor (Büchi labortechnik AG, Flawil, Switzerland). This dry concentrate was redissolved in one $\mathrm{mL}$ of water, filtered through a $0.45 \mu \mathrm{m}$ filter, and analyzed by high-performance anion exchange chromatography (HPAEC Dionex, Sunnyvale, Calif, USA). All processes were run in triplicate.

2.4. Fructooligosaccharides Analysis. FOS were separated on an HPLC-carbohydrate column PA1, Carbo Pack (Sunnyvale, Calif, USA) with a Dionex Bio LC series HPLC (Sunnyvale, Calif, USA) and pulsed amperometric detector (PAD). The gradient was established by mixing eluent A $(150 \mathrm{mM} \mathrm{NaOH})$ with eluent B $(500 \mathrm{mM}$ acetate- $\mathrm{Na}$ in $150 \mathrm{mM} \mathrm{NaOH}$ ) in two ways: System I: 0-1 minute, $25 \mathrm{mM}$; 1-2 minutes, 25-50 mM; 2-20 minutes, 50-200 mM, 20-22 minutes, $500 \mathrm{mM}$; 22-30, $25 \mathrm{mM}$; System II: 0-1 minute, $25 \mathrm{mM}$; 1-2 minute(s), 25-50 mM; 2-14 minutes, 50-500 mM, 14-22 minutes, 500 mM; 22-30, $25 \mathrm{mM}$. The established gradient of mixing eluent $\mathrm{A}$ with eluent $\mathrm{B}$ 
was $0-1$ minute, $95 \%$ A-5\% B; $1-2$ minute(s), $80 \%$ A20\% B; 2-20 minute(s), 60\% A-40\% B; 20-22 minute(s), $100 \%$ B, 22-30 minute(s), 95\% A-5\% B. The flow rate

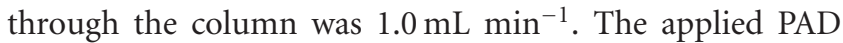
potentials for E1 (500 ms), E2 (100 milliseconds), and E3 (50 milliseconds) were $0.01,0.60$, and $-0.60 \mathrm{~V}$, respectively, and the output range was $1 \mu \mathrm{C}$. Samples $(25 \mu \mathrm{L})$ were injected using an autosampler (Intelligent autosampler, model AS4000, HITACHI Ltd, Tokyo, Japan). FOS were estimated by comparison with standards peaks.

2.5. Isolation of the Newly Formed FOS. The FOS were purified and isolated as shown in Figure 1. The stored onion bulbs ( $2 \mathrm{~kg}$ ) were extracted in $2000 \mathrm{~mL} \mathrm{70 \%} \mathrm{ethanol}$ containing a small amount of calcium carbonate $\left(0.5 \mathrm{~g} \mathrm{~L}^{-1}\right)$ in a boiling water bath. The treatment was repeated three times. The resulting extract was concentrated under vacuum at $35^{\circ} \mathrm{C}$ to $700 \mathrm{~mL}$, which, after addition of basic lead acetate, was allowed to stand over night. After the precipitate was removed by filtration, the filtrate was bubbled with hydrogen sulfide gas, and the resulting precipitate was filtered off. The solution was degassed, neutralized with $0.5 \mathrm{~N} \mathrm{NaOH}$, concentrated under vacuum, and lyophilized to give a white sugar powder $(86 \mathrm{~g})$. After the sugar powder was solubilized in water, the sugar solution was loaded onto a carbonCelite column $(6 \times 50 \mathrm{~cm})$ and eluted with water, $10 \%$, $15 \%$, and $30 \%$ ethanol successively. Because $10 \%$ ethanol eluate fraction contained di-, tri- and tetrasaccharides, the $10 \%$ ethanol fraction was re-chromatographed with carbonCelite column $(5 \times 75 \mathrm{~cm})$ by successive elution with water, 3 and 5\% ethanol. Saccharides 1 and 2 were eluted with $3 \%$ and $5 \%$ ethanol, respectively. Saccharides 1 and 2 fractions were concentrated under vacuum and lyophilized to give white powders, 25 and $10 \mathrm{mg}$, respectively. During these different purification processes, the fractions were identified using the method described earlier.

2.6. In Vitro Biosynthesis of Saccharides 1 and 2 by Purified Onion 6G-FFT. Saccharides 1 and 2 were synthesized in vitro using 6G-FFT purified from onion bulbs as described previously [15] and isolated as shown in Figure 2. A mixture of onion 6G-FFT ( $4 \mathrm{U}), 0.2 \mathrm{M}$ fructose, and $0.2 \mathrm{M}$ 1-kestose in McIlvaine buffer ( $\mathrm{pH} 5.5,60 \mathrm{~mL}$ ) was incubated at $30^{\circ} \mathrm{C}$ for 168 hours. After the reaction was stopped by heating at $100^{\circ} \mathrm{C}$ in a boiling water bath for 3 minutes, the reaction mixture was loaded on a carbon-Celite column $(6 \times$ $75 \mathrm{~cm}$ ) and eluted with water, 3 and $5 \%$ ethanol successively. Saccharides 1 and 2 were eluted with 3 and 5\% ethanol, respectively. Saccharides 1 and 2 fractions were concentrated under vacuum and lyophilized to give white powders, 182 and $53 \mathrm{mg}$, respectively,

2.7. Gas Liquid Chromatography (GLC) Analysis of Methanolyzates of Permethylated Saccharides 1 and 2. Methylation of the oligosaccharides was carried out by the method of Hakomori [27]. The permethylated saccharides were methanolysed by heating with $1.5 \%$ methanolic hydrochloric acid at $92^{\circ} \mathrm{C}$ for 10 minutes. The reaction mixture was treated with Amberlite IRA-410 (OH) to remove hydrochloric acid, and evaporated under vacuum to dryness. The resulting methanolysate was dissolved in a small volume of methanol and analyzed using gas chromatography (Hitachi 063 Type Gas Chromatograph Apparatus; stainless column $(3 \mathrm{~mm} \times$ $1 \mathrm{~m}$ ) packed with $15 \%$ butane-1, 4-diol succinate polyester on acid-washed Celite at $175^{\circ} \mathrm{C}$ and fitted with an FI detector, the carrier gas was nitrogen at the flow rate $40 \mathrm{~mL} \mathrm{~min}^{-1}$ ).

2.8. Matrix Assisted Laser Desorption Ionization Time of Flight Mass Spectrometry (MALDI-TOF-MS). MALDI-TOFF-MS: MALDI-TOF-MS spectra were measured using a ShimadzuKratos mass spectrometer (with KOMPACT Probe) in positive ion mode with $2.5 \%$-dihydroxybenzoic acid as a matrix. Ions were formed by a pulsed UV laser beam (nitrogen laser, $337 \mathrm{~nm}$ ). Calibration was done using nystose as an external standard.

2.9. Nuclear Magnetic Resonance (NMR) Measurements of Saccharides 1 and 2. Saccharides 1 ( $5 \mathrm{mg}$ ) and 2 (5 mg) were dissolved in $0.5 \mathrm{~mL} \mathrm{D}_{2} \mathrm{O}$. NMR spectra were recorded at room temperature with a Bruker AMX 500 spectrometer $\left({ }^{1} \mathrm{H}\right.$ $500 \mathrm{MH}_{Z},{ }^{13} \mathrm{C} 125 \mathrm{MH}_{Z}$ ) equipped with a $5 \mathrm{~mm}$ diameter $\mathrm{C} / \mathrm{H}$ dual (1D spectra) and TXI probe (2D spectra). Chemical shifts of ${ }^{1} \mathrm{H}\left(\delta_{\mathrm{H}}\right)$ and ${ }^{13} \mathrm{C}\left(\delta_{\mathrm{C}}\right)$ in ppm were determined relatively to the external standard of sodium $\left[2,2,3,3-{ }^{2} \mathrm{H}_{4}\right]$ 3 -(trimethylsilyl) propanoate in $\mathrm{D}_{2} \mathrm{O}\left(\delta_{\mathrm{H}} 0.00 \mathrm{ppm}\right)$ and 1,4 dioxane $\left(\delta_{\mathrm{C}} 67.40 \mathrm{ppm}\right)$ in $\mathrm{D}_{2} \mathrm{O}$, respectively. ${ }^{1} \mathrm{H}-{ }^{1} \mathrm{H}$ COSY and HSQC were obtained using gradient selected pulse sequences. The phase-sensitive HSQC-TOCSY spectra were determined with the sequence including inversion of directly resonance (IDR). HMBC spectra were obtained using the pulse sequences of CT-HMBC.

\section{Results and Discussion}

The formation of the two new FOS was observed in onion bulb tissues four weeks after harvesting and storage at $10^{\circ} \mathrm{C}$ as shown by the chromatograms (Figure $3(\mathrm{a})$ ). We noted that retention time of saccharides 1 and 2 was 6.87 and 9.52 minutes by HPAEC, respectively, and saccharide 1 was eluted after 1-kestose while saccharide 2 was eluted after nystose (4a). When synthesized in vitro by 6G-FFT, saccharides 1 and 2 also showed similar retention times, 6.89 and 9.54 minutes, and were eluted after the same FOS, that is, 1kestose and nystose (Figure 3(b)). We also found at first that the presence of 1-kestose as sole substrate did not initiate the reaction, while in the presence of fructose and 1-kestose, the reaction was initiated. In vivo, the fructose is provided by the hydrolysis of other FOS in a reaction catalyzed by 1-FEH (fructan:fructan 1-exohydrolase).

The saccharides 1 and 2 isolated from both onion tissues and enzyme reaction were subjected to GLC and NMR analyses (Tables 1, 2 and Figure 4). The methanolyzates of saccharides 1 and 2 showed four peaks corresponding to methyl 1,3,4,6-tetra-o-methyl fructoside and methyl 3,4,6tri-o-methylfructosideon on GLC chromatograms, respectively. From the findings above, saccharides 1 and 2 were 


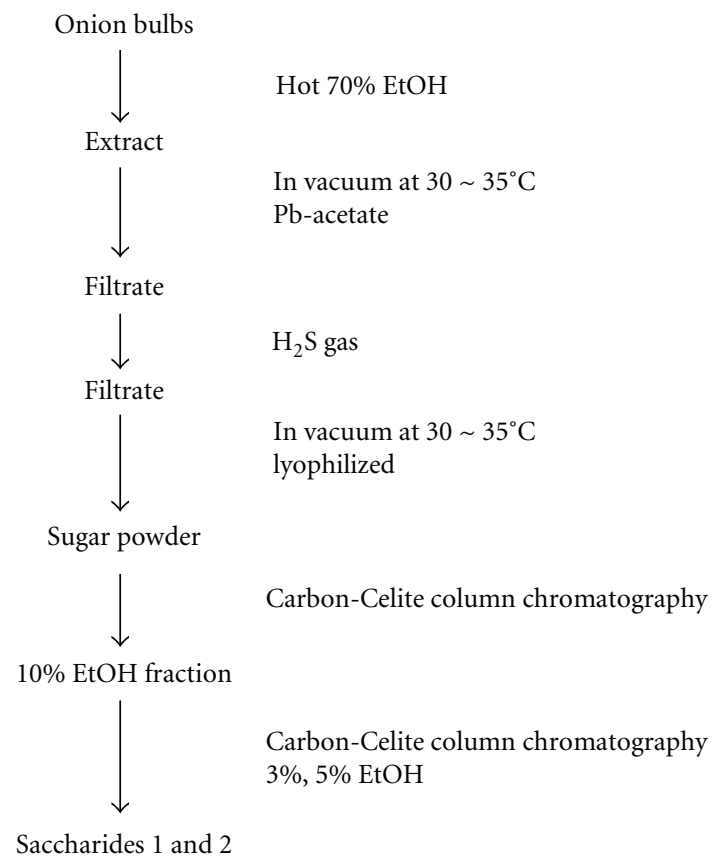

FIGURE 1: Isolation procedure of saccharides 1 and 2 from onion bulbs stored one month at $10^{\circ} \mathrm{C}$.

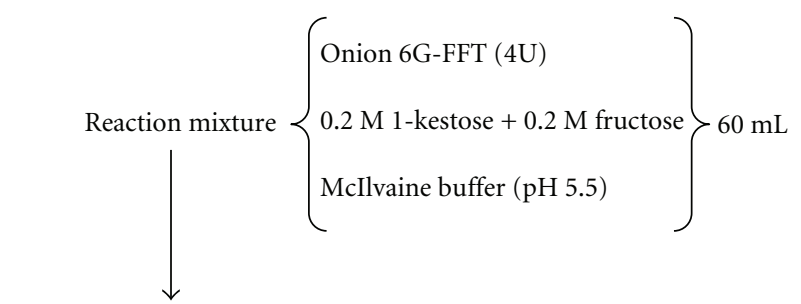

Incubation at $30^{\circ} \mathrm{C}$ for $168 \mathrm{~h}$.

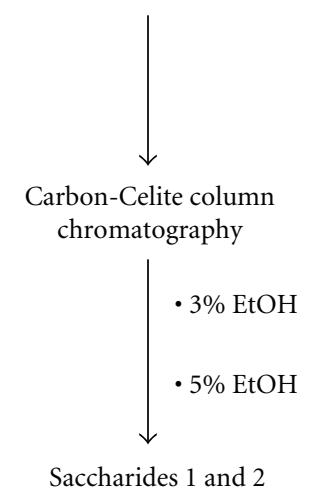

FIGURE 2: Isolation of saccharides 1 and 2 synthesized by purified onion 6G-FFT in vitro.

TABLE 1: GLC analysis of methanolyzates of permethylated saccharides 1 and 2 isolated from onion bulbs.

\begin{tabular}{lcccc}
\hline \multirow{2}{*}{ Methanolyzate from permethylated } & \multicolumn{3}{c}{ Relative retention time* } \\
& Methyl 1,3,4,6-tetra- $O$-methyl-D-fructoside & Methyl 3,4,6-tri-O-methyl-D-fructoside \\
Saccharide 1 & 1.03 & 1.25 & 2.60 & 3.89 \\
Saccharide 2 & 1.03 & 1.26 & 2.67 & 3.96 \\
\hline
\end{tabular}

${ }^{*}$ Retention time of methyl 2,3,4,6-tetra-O-methyl- $\beta$-D-glucoside $=1$. 


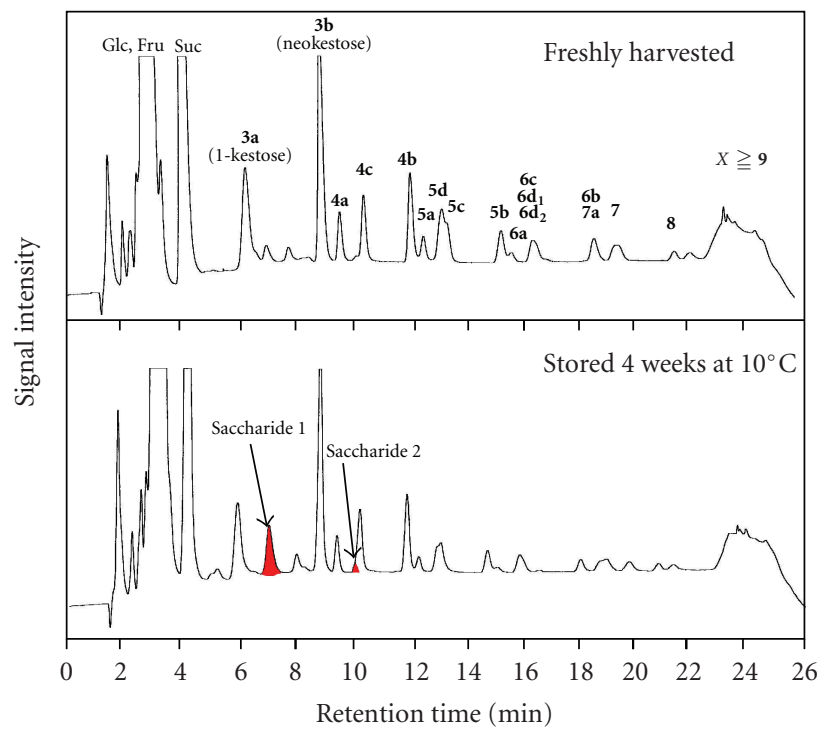

(a)

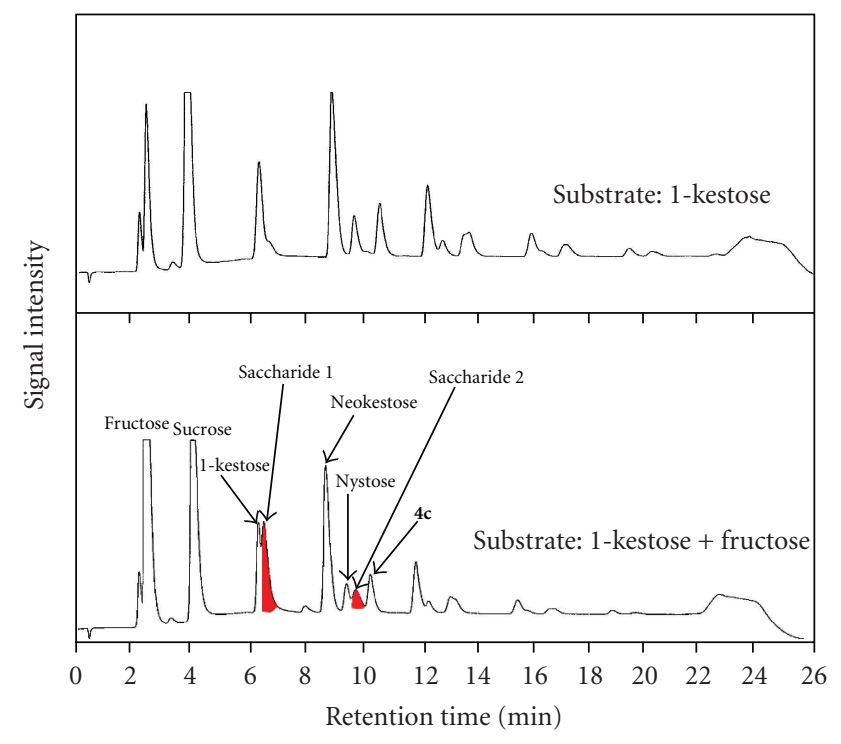

(b)

FIGURE 3: HPAEC charts of sacchaides 1 and 2; (a) chart of saccharides 1 and 2 extracted and purified from tissues of onion bulbs stored one month at $10^{\circ} \mathrm{C},(\mathrm{b})$ : saccharides 1 and 2 synthesized in vitro by purified 6G-FFT of onion.
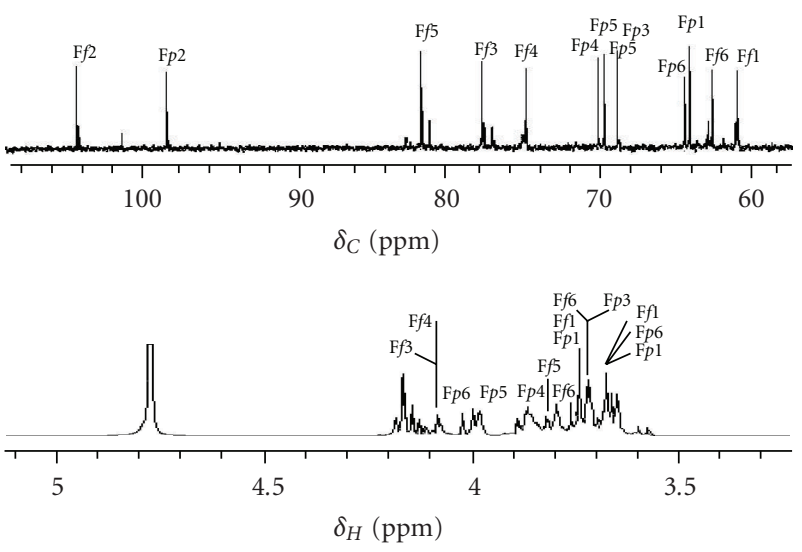

(a)
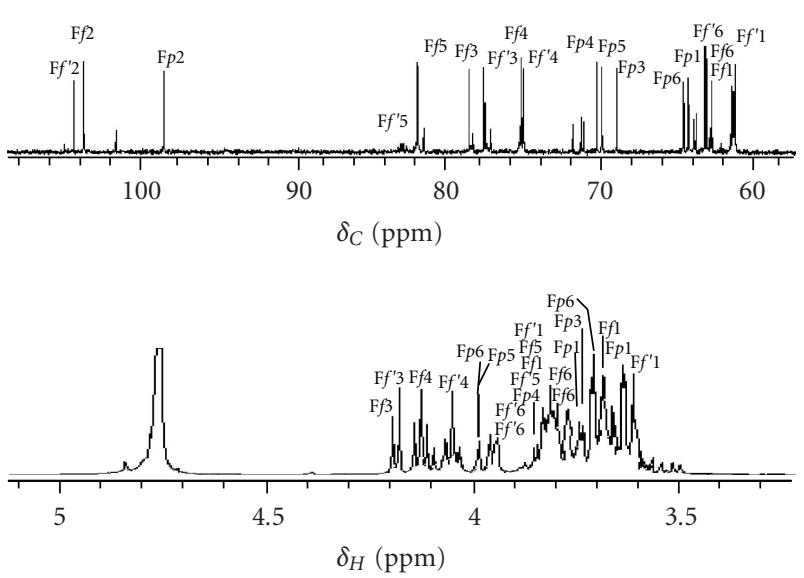

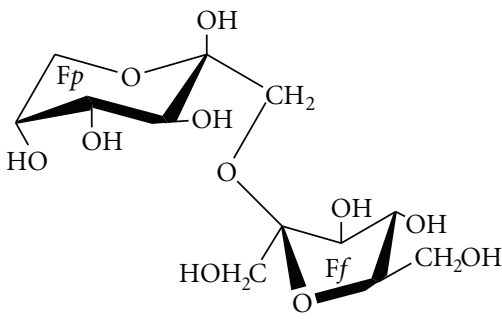

Saccharide 1

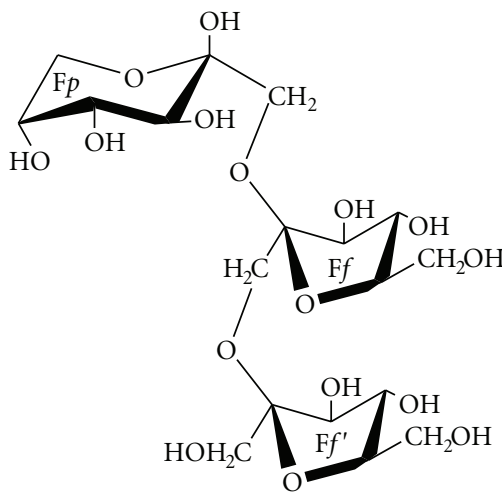

Saccharide 2

(b)

Figure $4:{ }^{13} \mathrm{C}$ - and ${ }^{1} \mathrm{H}-\mathrm{NMR}$ spectra of saccharides 1 and 2. Ff; fructofuranosyl, Fp; fructopyranosyl. 


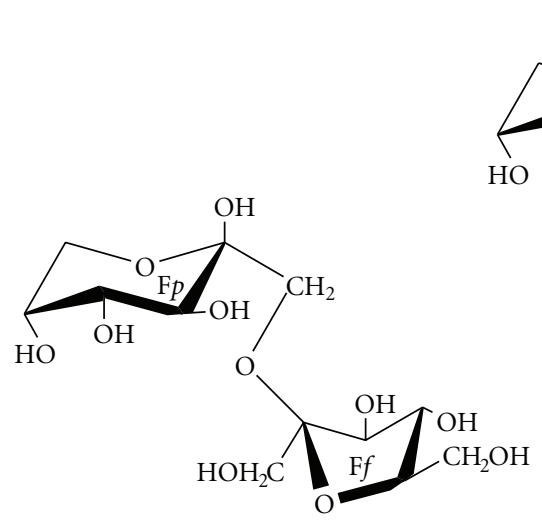

Saccharide 1

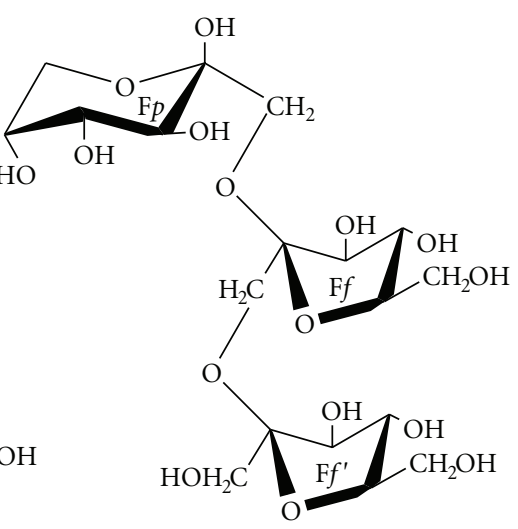

Saccharide 2

Figure 5: The structure of saccharides 1 and 2. F $f$; fructofuranosyl, Fp; fructopyranosyl.
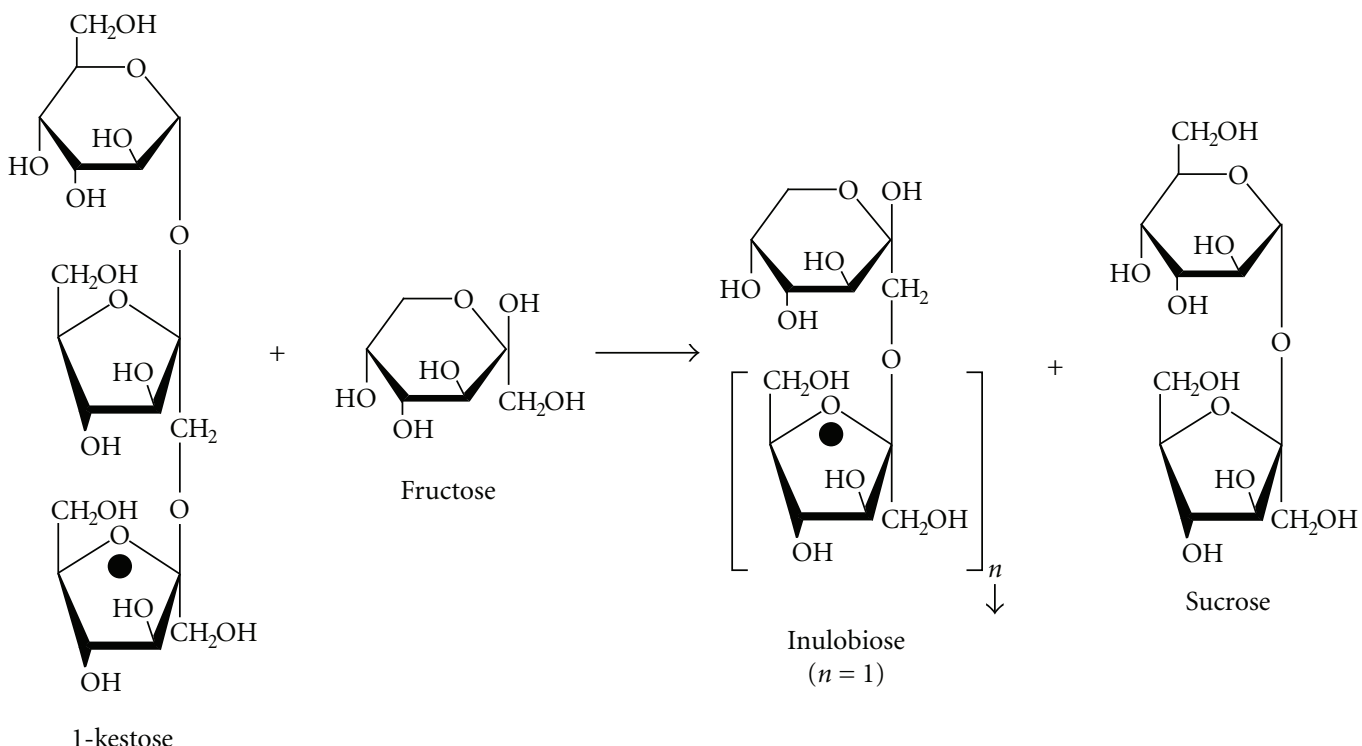

Sucrose $(n=1)$

FIGURE 6: In vitro biosynthesis of inulobiose (saccharide 1) by purified onion 6G-FFT (full black circle indicate the transferred fructose moiety).

confirmed to be fructosyl- $(2 \rightarrow 1)$-fructose and fructosyl$(2 \rightarrow 1)$-fructosyl- $(2 \rightarrow 1)$-fructose.

From the chemical shift values and the relative intensities of the ${ }^{13} \mathrm{C}$ and ${ }^{1} \mathrm{H}$ NMR signals of the $\mathrm{C} 2$ atoms, it was concluded that saccharides 1 and 2 contain one reducing fructopyranosyl residue. The presence of typical signals for C2, C3, C4, and C5 atoms of fructopyranose residues shows the reducing end lacks $6-\mathrm{OH}$ group. The spectra of the analyses confirmed the presence of two different structures: one structure composed by two fructose moieties and linked by $\beta(2 \rightarrow 1)$ linkage and identified as inulobiose $[\beta-\mathrm{D}$ fructofuranosyl- $(2 \rightarrow 1)$ - $\beta$-D-fructopyranose], while saccharide 2 consists of three units of fructose linked by $\beta(2 \rightarrow$ $1)$ linkage and was inulotriose $[\beta$-D-frucofuranosyl- $(2 \rightarrow 1)$ $\beta$-D-fructofuranosyl- $(2 \rightarrow 1)-\beta$-D-fructopyranse] (Figure 5 ). The spectra also did show that $70-80 \%$ of the terminal (reducing) fructose residue of the two saccharides, inulobiose and inulotriose, have the pyranose form, while 20 to $30 \%$ have furanose form.

The two saccharides were subjected to some chemical property analyses as shown in Table 3 . The purified saccharides 1 and 2 from onion bulb tissues had R-sucrose (retention time of the saccharide to retention time of sucrose) values of 1.55 and 2.15 by HPAEC, a reducing terminal, a reducing sugar-to-fructose ratio of 0.5 and 0.3 , and a degree of polymerization determined (DP) by MALDITOF-MS of 2 and 3, respectively. Comparatively, the in vitro biosynthesized saccharides 1 and 2 showed similar chemical properties and exactly the same values for these parameters were noted (Table 3 ). However, due to the small amount of saccharides 1 and 2 obtained after purification (25 and $10 \mathrm{mg}$, resp.), it was not possible to determine the melting point of these two molecules. The suggested reaction of biosynthesis of these two saccharides is shown 
TABLE 2: GLC analysis of methanolyzates of permethylated saccharides 1 and 2 synthesized by purified from G-FFT from onion bulbs.

\begin{tabular}{lcccc}
\hline \multirow{2}{*}{ Methanolyzate from permethylated } & \multicolumn{4}{c}{ Relative retention time* } \\
& Methyl 1,3,4,6-tetra- $O$-methyl-D-fructoside & \multicolumn{2}{c}{ Methyl 3,4,6-tri- $O$-methyl-D-fructoside } \\
Saccharide 1 & 1.04 & 1.25 & 2.59 & 3.86 \\
Saccharide 2 & 1.03 & 1.26 & 2.66 & 3.94 \\
\hline
\end{tabular}

*Retention time of methyl 2,3,4,6-tetra- $O$-methyl- $\beta$-D-glucoside $=1$.

TABLE 3: Some chemical properties of saccharides 1 and 2 (a) isolated from onion bulb tissues stored one month at $10^{\circ} \mathrm{C}$, and (b) synthesized by purified 6G-FFT from onion.

\begin{tabular}{lccccc}
\hline \multicolumn{5}{c}{ (a) Saccharides isolated from onion bulns } \\
\hline Saccharide & R-sucrose value & Reducing power & Molar ratio in the hydrolyzate: Red. Sugar/fructose & TOF-MS m/z & DP \\
1 & 1.55 & + & 0.5 & 365 & 2 \\
2 & 2.15 & + & (b) Saccharides synthesized by purified 6G-FFT from onion & 527 & 3 \\
\hline & & Reducing power & Molar ratio in the hydrolyzate: Red. Sugar/fructose & TOF-MS m/z & DP \\
\hline Saccharide & R-sucrose value* & Rer & 0.5 & 365 & 2 \\
1 & 1.56 & + & 0.3 & 527 \\
2 & 2.15 & + & & 3 \\
\hline
\end{tabular}

${ }^{*} \mathrm{R}$-sucrose $=$ retention time of the saccharide to retention time of sucrose.

in Figure 6. 1-Kestose is the donor of the fructosyl unit, and the fructopyranosyl the acceptor. The analyses showed that the acceptor fructose is under the pyranosyl form because in aqueous solution, free fructose has almost pyranose form.

As plausible hypothesis, this biosynthesis might be induced by the quantity of free fructose in the form of fructopyranose found in the vacuoles resulting from the breakdown of FOS. Because of the continuous breakdown and release of fructose by the hydrolyzing activity of 1-FEH during storage of onion bulbs [28], this free fructose is used as a substrate for the synthesis of these new saccharides avoiding high accumulation of fructose which could be detrimental to the metabolic activities in the vacuoles by increasing the osmotic pressure of these organelles leading to the slowing down of the metabolic activities. This fact could be emphasized by the high water content of onion tissues (more than $85 \%$ ), and the high 1-FEH activity observed preceding the onset of sprouting [28]. This high activity of 1-FEH reported previously [28] coincides well with the appearance of the two saccharides which are observed after at least one-moth storage. The synthesis carried out in vitro showed that the reaction is quite linear but faster during the first 100 hours slowing down after this (Figure 7). This pattern is regular and showed that reaction occurs without apparent inhibition, mainly caused by sucrose which is known to inhibit many enzymatic reactions when its concentration is high [16].

The formation of fructosyl-saccharides or fructo-inulinoses has been reported first in chicory during its forcing after a growth and storage [29]. The authors observed some changes which consisted of a significant increase in fructose concentration; an increase in the concentration of low degree of polymerization (DP) fructans; a decrease in high DP fructans; and the appearance new fructans without terminal glucose. Later, Van den Ende et al. [17] obtained three different fructose oligosaccharides, inulobiose (DP 2),

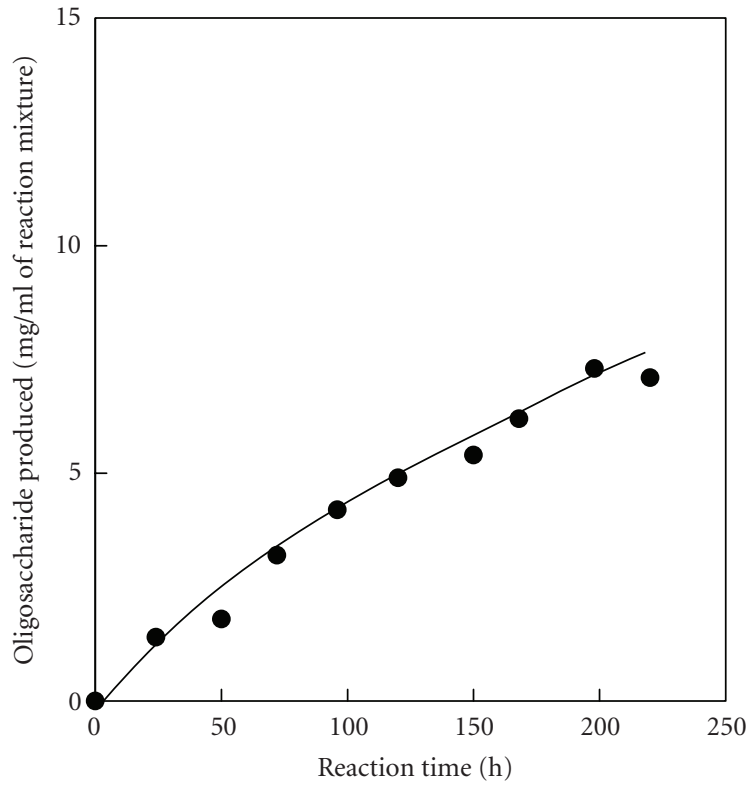

FIGURE 7: Time course of formation of saccharide 1 catalyzed by $6 \mathrm{G}-\mathrm{FFT}$ in vitro.

inulotriose (DP 3), and inulotetraose (DP 4), by in vitro biosynthesis using purified chicory root fructan:fructan fructosyl transferase (FFT) with inulin as a donor and fructose as an acceptor. These newly produced fructooligosaccharides were also isolated from chicory roots [18]. Similar inulon-ose series of DP 2 and DP 3 of fructose polymers were identified in both sprouted and unsprouted onion bulbs after two-month storage [19]. However, although the degree of polymerization was determined, these authors did not report indeed the structural conformation of the fructosyl terminal of these isolated saccharides as reported in the present investigation. 
Although from the chemical point of view we could suggest that the reaction would be triggered by the accumulation of fructose released from the hydrolysis of fructans and/or by the pyranosyl form of the free fructose in the vacuoles, the physiological process inducing this reaction is unclear and enigmatic. We can speculate on the fact that the formation of these short-chain inulibiose or inulintriose is initated by the high accumulation of fructose in the vacuoles and the possible unbalance and osmotic pressure created by this accumulation. Thus, the synthesis of these two new saccharides could play either a role of "balance" between the levels of the different high DP FOS $(\geq 5)$ and/or a "buffer reaction" between the different tri- and tetrasaccharides, because of the low rate of fructose utilization in the metabolism. This speculation would be supported by further work on one hand the hydrolysis kinetic parameters of these saccharides and compare them with the parameters of other FOS studied previously, and on the other hand the rapid exhausting of high DP FOS $(5 \sim 12)$, while low DP FOS $(3 \sim 4)$ decrease less during the first two months of storage $[11,12]$.

In conclusion, the in vitro biosynthesis of new short saccharides composed by fructose molecules seem to occur under specific conditions such as forcing (chicory) or storage (chicory and onion). However, this reaction is unclear and the questions raised are: (i) what triggers this reaction?, (ii) which physiological is behind this reaction?, and (iii) what are the roles of these newly formed compounds? Thus, a lot remains to be investigated in order to answer these questions and understand better the complex metabolism of fructans in plants.

\section{References}

[1] B. Darbyshire and R. J. Henry, "Differences in fructan content and synthesis in some Allium species," New Phytologist, vol. 87, no. 2, pp. 249-256, 1981.

[2] N. Benkeblia, P. Varoquaux, N. Shiomi, and H. Sakai, "Storage technology of onion bulbs c.v. Rouge Amposta: effects of irradiation, maleic hydrazide and carbamate isopropyl, N-phenyl (CIP) on respiration rate and carbohydrates," International Journal of Food Science \& Technology, vol. 37, no. 2, pp. 169175, 2002.

[3] B. Darbyshire, "Changes in the carbohydrate content of onion bulbs stored for various times at different temperatures," Journal of Horticultural Science, vol. 53, no. 2, pp. 195-201, 1978.

[4] N. Benkeblia, S. Onodera, and N. Shiomi, "Effect of gamma irradiation and temperature on fructans (fructo-oligosaccharides) of stored onion bulbs Allium cepa L," Food Chemistry, vol. 87, no. 3, pp. 377-382, 2004.

[5] N. Shiomi, S. Onodera, and H. Sakai, "Fructo-oligosaccharide content and fructosyltransferase activity during growth of onion bulbs," New Phytologist, vol. 136, no. 1, pp. 105-113, 1997.

[6] J. E. Spiegel, R. Rose, P. Karabell, V. H. Frankos, and D. F. Schmitt, "Safety and benefits of fructooligosaccharides as food ingredients," Food Technology, vol. 1, no. 1, pp. 85-89, 1994.

[7] G. Flamm, W. Glinsmann, D. Kritchevsky, L. Prosky, and M. Roberfroid, "Inulin and oligofructose as dietary fiber: a review of the evidence," Critical Reviews in Food Science and Nutrition, vol. 41, no. 5, pp. 353-362, 2001.

[8] A. Franck, "Technological functionality of inulin and oligofructose," British Journal of Nutrition, vol. 87, supplement 2, pp. S287-S291, 2002.

[9] C. Blecker, C. Fougnies, J.-C. Van Herck, J.-P. Chevalier, and M. Paquot, "Kinetic study of the acid hydrolysis of various oligofructose samples," Journal of Agricultural and Food Chemistry, vol. 50, no. 6, pp. 1602-1607, 2002.

[10] C. L'Homme, M. Arbelot, A. Puigserver, and A. Biagini, "Kinetics of hydrolysis of fructooligosaccharides in mineralbuffered aqueous solutions: influence of $\mathrm{pH}$ and temperature," Journal of Agricultural and Food Chemistry, vol. 51, no. 1, pp. 224-228, 2003.

[11] N. Benkeblia, N. Takahashi, K. Ueno, S. Onodera, and N. Shiomi, "Tetra- and penta-fructooligosaccharide (FOS) isomers assessment in onion bulb tissues: effect of temperature and storage time," Tetrahedron: Asymmetry, vol. 16, no. 1, pp. 33-37, 2005.

[12] N. Benkeblia and N. Shiomi, "Hydrolysis kinetic parameters of DP 6, 7, 8, and 9-12 fructooligosaccharides (FOS) of onion bulb tissues. Effect of temperature and storage time," Journal of Agricultural and Food Chemistry, vol. 54, no. 7, pp. 2587-2592, 2006.

[13] N. Benkeblia, S. Onodera, and N. Shiomi, "Effect of temperature and storage time on fructosyltransferase activities (1FFT and 6G-FFT) in onion bulb tissues," Acta Agriculturae Scandinavica B, vol. 53, no. 4, pp. 211-214, 2003.

[14] T. Ritsema, J. Joling, and S. Smeekens, "Patterns of fructan synthesized by onion fructan: fructan 6G-fructosyltransferase expressed in tobacco BY2 cells-is fructan: fructan 1fructosyltransferase needed in onion?" New Phytologist, vol. 160, no. 1, pp. 61-67, 2003.

[15] M. Fujishima, H. Sakai, K. Ueno, et al., "Purification and characterization of a fructosyltransferase from onion bulbs and its key role in the synthesis of fructo-oligosaccharides in vivo," New Phytologist, vol. 165, no. 2, pp. 513-524, 2005.

[16] W. Wagner, A. Wiemken, and P. Matile, "Regulation of fructan metabolism in leaves of barley (Hordeum vulgare L. cV Gerbel)," Plant Physiology, vol. 81, no. 2, pp. 444-447, 1986.

[17] W. Van den Ende, J. De Roover, and A. Van Laere, "In vitro synthesis of fructofuranosyl-only oligosaccharides from inulin and fructose by purified chicory root fructan: fructan fructosyl transferase," Physiologia Plantarum, vol. 97, no. 2, pp. 346352, 1996.

[18] J. W. Timmermans, T. M. Slaghek, M. Iizuka, W. Van den Ende, J. De Roover, and A. Van Laere, "Isolation and structural analysis of new fructans produced by chicory," Journal of Carbohydrate Chemistry, vol. 20, no. 5, pp. 375-395, 2001.

[19] M. K. Ernst, N. J. Chatterton, P. A. Harrison, and G. Matitschka, "Characterization of fructan oligomers from species of the genus Allium L.," Journal of Plant Physiology, vol. 153, no. 1-2, pp. 53-60, 1998.

[20] H. Takeda, K. Sato, S. Kinoshita, and H. Sasaki, "Production of 1-kestose by Scopulariopsis brevicaulis," Journal of Fermentation and Bioengineering, vol. 77, no. 4, pp. 386-389, 1994.

[21] N. Shiomi, J. Yamada, and M. Izawa, "Isolation and identification of fructo-oligosaccharides in roots of asparagus (Asparagus officinalis L.)," Agricultural and Biological Chemistry, vol. 40, no. 3, pp. 567-575, 1976.

[22] N. Shiomi, J. Yamada, and M. Izawa, "A novel pentasaccharide in the roots of asparagus (Asparagus officinalis L.)," Agricultural and Biological Chemistry, vol. 43, no. 6, pp. 1375-1377, 1979. 
[23] N. Shiomi, "Two novel hexasaccharides from the roots of Asparagus officinalis," Phytochemistry, vol. 20, no. 11, pp. 2581-2583, 1981.

[24] D. H. Lewis, "Nomenclature and diagrammatic representation of oligomeric fructans- a paper for discussion," New Phytologist, vol. 124, no. 4, pp. 583-594, 1993.

[25] A. L. Waterhouse and N. J. Chatterton, "Glossary of fructan terms," in Science and Technology of Fructans, M. Suzuki and N. J. Chatterton, Eds., pp. 1-7, CRC Press, Boca Raton, Fla, USA, 1993.

[26] N. Shiomi, "Content of carbohydrate and activities of fructosyltransferase and invertase in asparagus roots during fructooligosaccharide- and fructopolysaccharide accumulating season," New Phytologist, vol. 122, no. 3, pp. 421-432, 1992.

[27] S. A. Hakomori, "Rapid permethylation of glycolipid, and polysaccharide catalysed by methylsulfinyl carbanion in dimethyl sulfoxide," Journal of Biochemistry, vol. 55, no. 2, pp. 205-208, 1964.

[28] N. Benkeblia, S. Onodera, and N. Shiomi, "Variation in 1fructo-exohydrolase (1-FEH) and 1-kestose-hydrolysing (1$\mathrm{KH}$ ) activities and fructo-oligosaccharide (FOS) status in onion bulbs. Influence of temperature and storage time," Journal of the Science of Food and Agriculture, vol. 85, no. 2, pp. 227-234, 2005.

[29] W. Van den Ende, A. Mintiens, H. Speleers, A. A. Onuoha, and A. Van Laere, "The metabolism of fructans in roots of Cichorium intybus during growth, storage and forcing," New Phytologist, vol. 132, no. 4, pp. 555-563, 1996. 


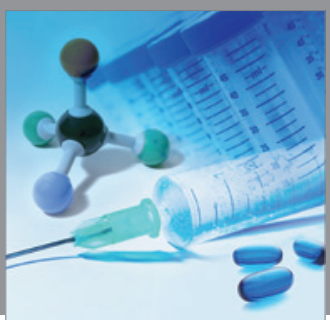

International Journal of

Medicinal Chemistry

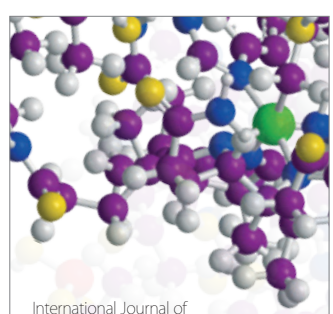

Carbohydrate Chemistry

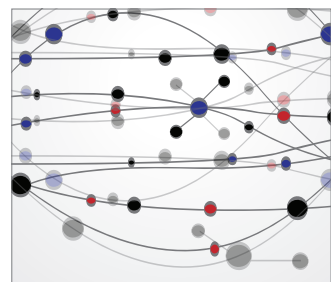

The Scientific World Journal
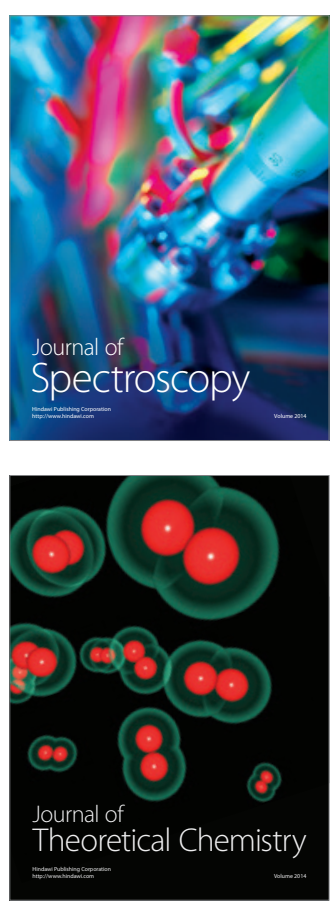
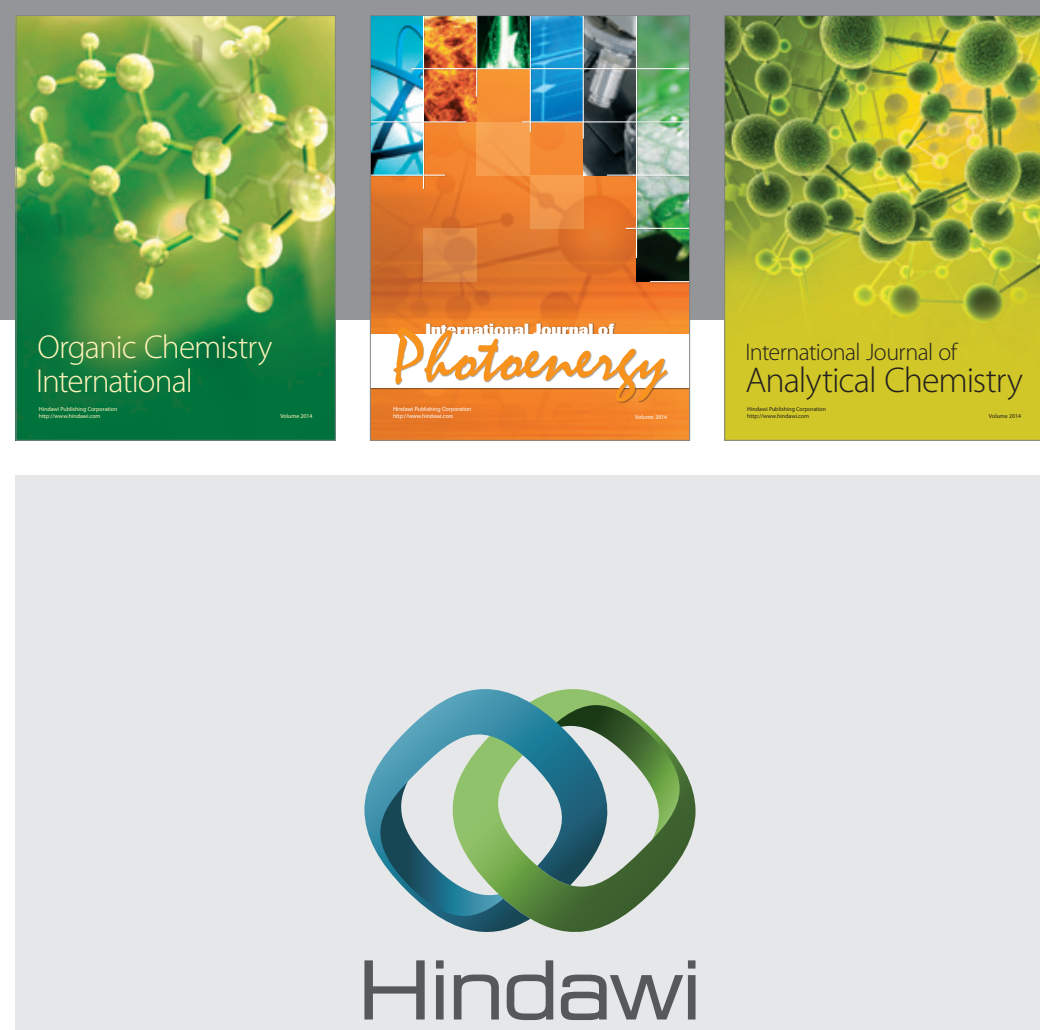

Submit your manuscripts at

http://www.hindawi.com
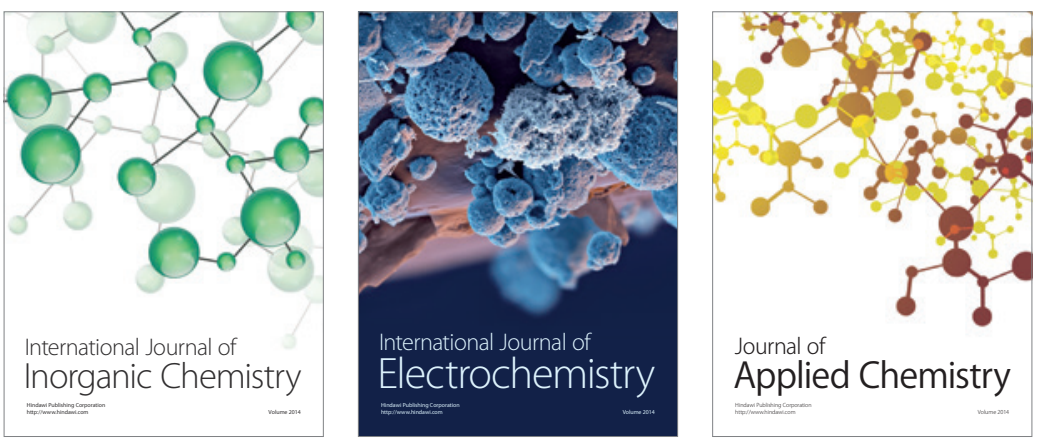

Journal of

Applied Chemistry
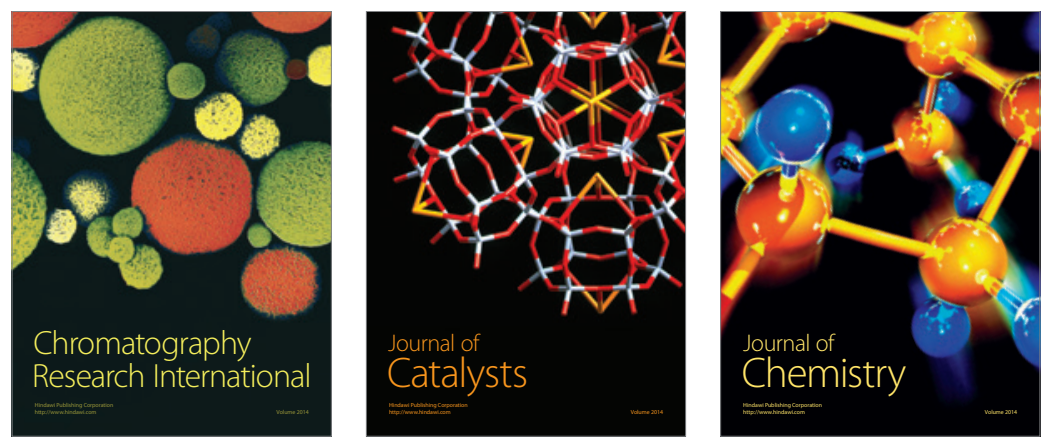
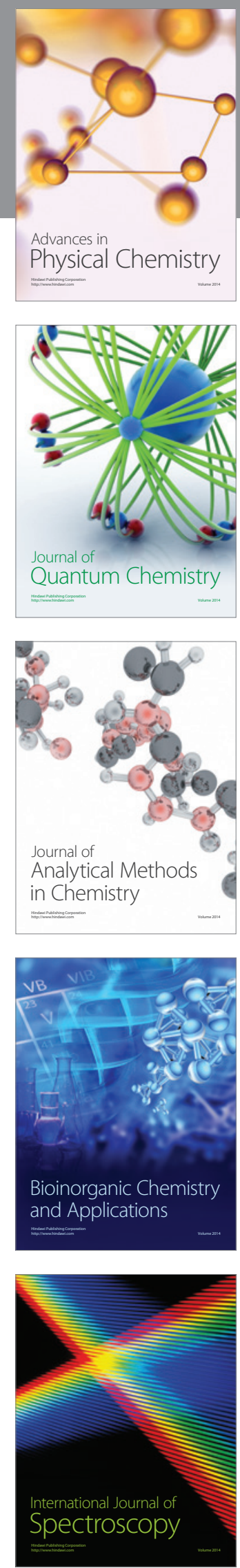